\title{
Nanoengineered Templated Polymer Particles: Navigating the Biological Realm
}

Jiwei Cui, ${ }^{\dagger}$ Joseph J. Richardson, ${ }^{\dagger, \ddagger}$ Mattias Björnmalm, ${ }^{\dagger}$ Matthew Faria, ${ }^{\dagger, \S}$ and Frank Caruso ${ }^{\dagger, *}$

${ }^{\dagger}$ Australian Research Council (ARC) Centre of Excellence in Convergent Bio-Nano Science and Technology, and the Department of Chemical and Biomolecular Engineering, the University of Melbourne, Parkville, Victoria 3010, Australia.

${ }^{\S}$ ARC Centre of Excellence in Convergent Bio-Nano Science and Technology, and the Systems Biology Laboratory, Melbourne School of Engineering, the University of Melbourne, Parkville, Victoria 3010, Australia.

${ }^{\ddagger}$ Present address: CSIRO Manufacturing Flagship, CSIRO Private Bag 10, Clayton South, Victoria 3169, Australia. 


\section{CONSPECTUS:}

Nanoengineered materials offer tremendous promise for developing the next generation of therapeutics. We are transitioning from simple research questions, such as "can this particle eradicate cancer cells?" to more sophisticated ones like “can we design a particle to preferentially deliver cargo to a specific cancer cell type?” These developments are poised to usher in a new era of nanoengineered drug delivery systems.

We primarily work with templating methods for engineering polymer particles, and investigate their biological interactions. Templates are scaffolds that facilitate the formation of particles with well-controlled size, shape, structure, stiffness, stability, and surface chemistry. In the past decade, breakthroughs in engineering new templates, combined with advances in coating techniques, including layer-by-layer (LbL) assembly, surface polymerization, and metal-phenolic network (MPN) coordination chemistry, have enabled particles with specific physicochemical properties to be engineered. While materials science offers an ever-growing number of novel synthesis techniques, a central challenge of therapeutic delivery has become understanding how nanoengineered materials interact with biological systems. Increased collaboration between chemists, biologists, and clinicians has resulted in a vast research output on bio-nano interactions. Our understanding of cell-particle interactions has grown considerably, but conventional in vitro experimentation provides limited information, and understanding how to bridge the in vitro/in vivo gap is a continuing challenge. As has been demonstrated in other fields, there is now a growing interest in applying computational approaches to advance this area. A considerable knowledge base is now emerging, and with it comes new and exciting opportunities that are already being capitalized on through the translation of materials into the clinic. 
In this Account, we outline our perspectives gained from a decade of work at the interface between polymer particle engineering and bio-nano interactions. We divide our research into three areas: (i) biotrafficking, including cellular association, intracellular transport, and biodistribution; (ii) biodegradation, and how to create controlled, responsive release of therapeutics; and (iii) applications, including drug delivery, controlling immunostimulatory response, biosensing, and microreactors. There are common challenges in these areas for groups developing nanoengineered therapeutics.

A key "lesson-learned" has been the considerable challenge of staying informed about the developments relevant to this field. There are a number of reasons for this, most notably the interdisciplinary nature of the work, the large numbers of researchers and research outputs, and the limited standardization in technique nomenclature. Additionally, a large body of work is being generated with limited central archiving, other than vast non-specific databases, such as Web of Science and PubMed. To help address these points, we have created a web-based tool to organize our past, present, and future work [Bio-nano research knowledgebase. http://bionano.eng.unimelb.edu.au/knowledge_base/ (accessed May 2, 2016)]. This tool is intended to serve as a first step toward organizing results in this large, complex area. We hope that this will inspire researchers, both in generating new ideas, and also in collecting, collating and sharing their experiences to guide future research. 


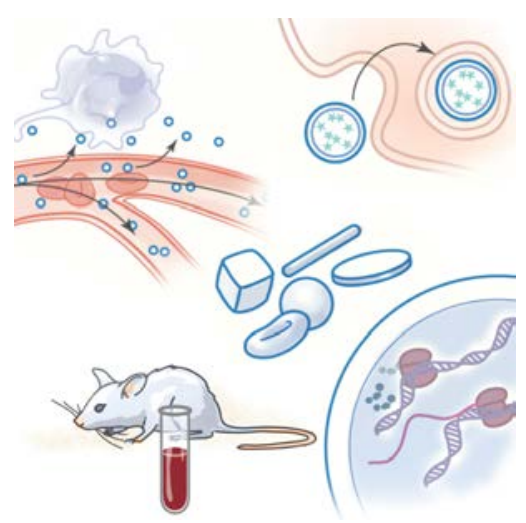




\section{INTRODUCTION}

The central goal of particle-based therapeutics is to increase therapeutic efficacy while decreasing side effects. Recently, there has been considerable research into the interactions between biological systems and nanostructured materials (bio-nano interactions), which will guide the engineering of the next generation of therapeutic vehicles. ${ }^{1,2}$ Nanotechnology is an important bridge between engineering and biotechnology, and has advanced the design and characterization of particles for biomedical applications. ${ }^{3,4}$ As a result, a range of delivery systems, including numerous types of polymer particles, have been generated over the past three decades. Importantly, the physicochemical properties of these polymer particles, such as the size, shape, rigidity, structure, and surface chemistry, are crucial in guiding our understanding of bionano interactions and related biomedical applications. ${ }^{5,6}$

The nanotechnology-driven templating method for the fabrication of polymer particles can be achieved via coating, growing, or infiltrating polymers on the surfaces or into the pores of a template, followed by polymer stabilization or cross-linking, and finally template removal. Templating is a versatile technique that provides a means of controlling the physicochemical properties of the resultant polymer particles. For example, size and shape are usually dependent on the morphology of the template; ${ }^{7,8}$ rigidity can be controlled by cross-linking density or polymer layer thickness; ${ }^{9,10}$ hollow or network structures can be tuned by using solid or porous templates; ${ }^{11,12}$ and the surface chemistry can be varied depending on polymer composition and post-functionalization. ${ }^{13}$ Different techniques, such as layer-by-layer (LbL) assembly, ${ }^{14}$ surface polymerization, $^{15}$ and mesoporous silica (MS) templating ${ }^{16}$ have been developed to engineer these physicochemical properties. 
This account will focus on our recent endeavors in studying bio-nano interactions governed by the physicochemical properties of templated polymer particles. In the past 10 years we have transitioned from focusing on particle design to focusing on both in vitro and in vivo studies (Figure 1). The first section includes the effects of physicochemical properties of particles on cellular association, including cellular binding and uptake, intracellular trafficking, biodistribution, and pharmacokinetics. Degradation is crucial for particles in controlled therapeutic delivery, therefore, the second section highlights particle degradability due to biological stimuli (e.g., pH, redox potential, and enzymes) during microenvironment changes in the cellular uptake process. In the final section, we discuss applications of templated polymer particles in drug and vaccine delivery, as well as in the design of sensors and bioreactors.

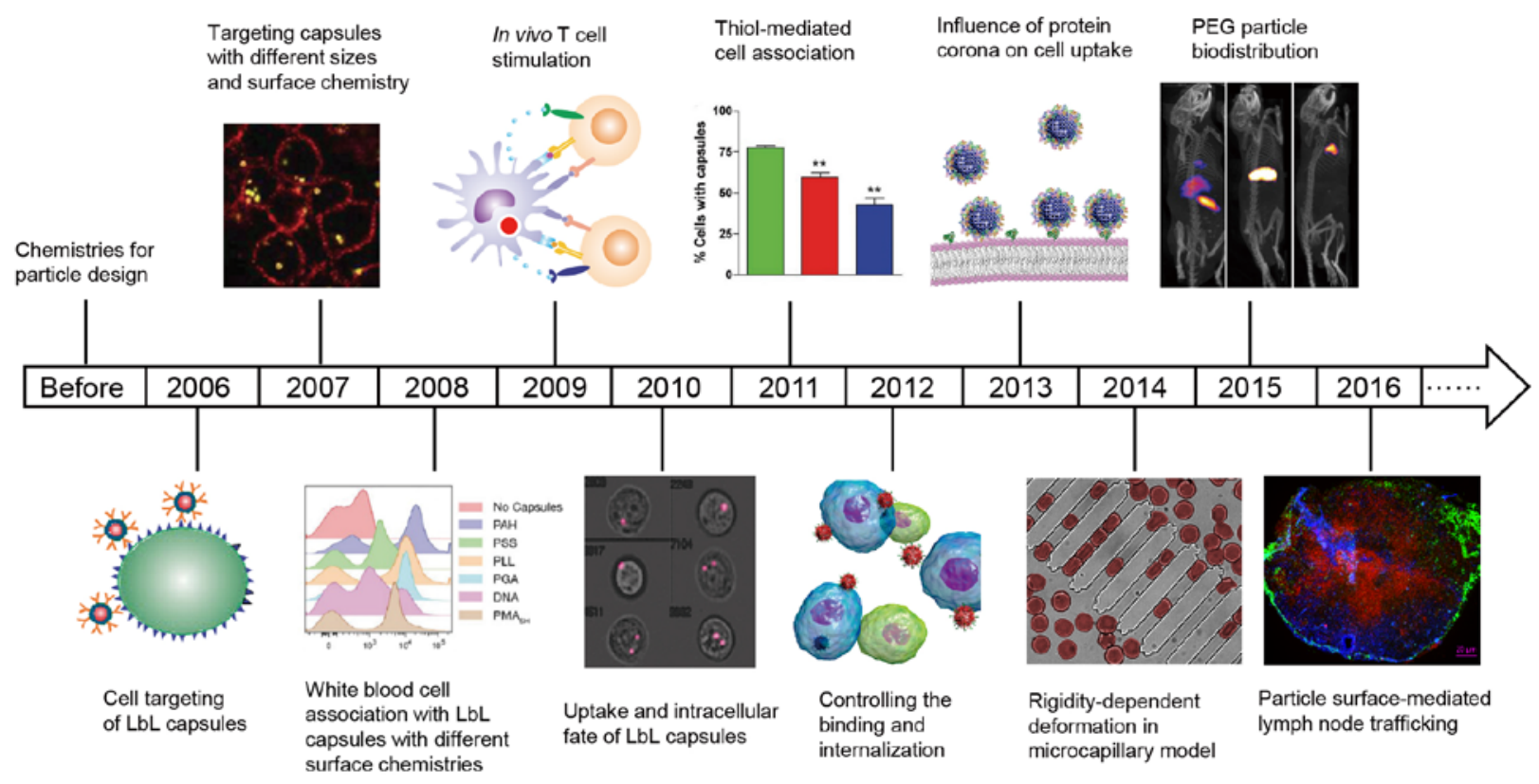

Figure 1. Timeline providing examples of bio-nano interactions studied using particles assembled through templating methods in the Caruso group. Parts of this figure are adapted with 
permission from references 7,17-25. Copyright 2006, 2008, 2011, 2014, 2016 Wiley-VCH, and copyright 2007, 2010, 2012, 2013, 2015 American Chemical Society.

\section{IN VITRO AND IN VIVO INTERACTIONS}

Bio-nano interactions of polymer particles at the cellular level include cellular binding, cellular uptake, and intracellular trafficking, which are all influenced by the physicochemical properties of particles (Figure 2). Particle size and shape have been widely reported to influence uptake pathways and intracellular trafficking in vitro. ${ }^{2,26}$ Additionally, size and shape influence particle flow, margination, and adhesive properties in blood vessels. ${ }^{27}$ Particle stiffness is another complex factor that influences both cellular association and uptake. ${ }^{28}$ The precise effect of varying stiffness depends on cell type, surface chemistry, size, and geometry. ${ }^{29}$ For instance, we observed higher cell binding and uptake with soft, hollow particles, which we attribute to their deformation upon contact with the cell membrane, leading to higher contact area. ${ }^{10,30}$ In contrast to this, others have reported higher uptake when using stiffer particles. ${ }^{31}$ It is also important to note that terms such as elasticity, hardness, rigidity, and flexibility are widely and often interchangeably used in the literature, though they are not equivalent. ${ }^{29}$
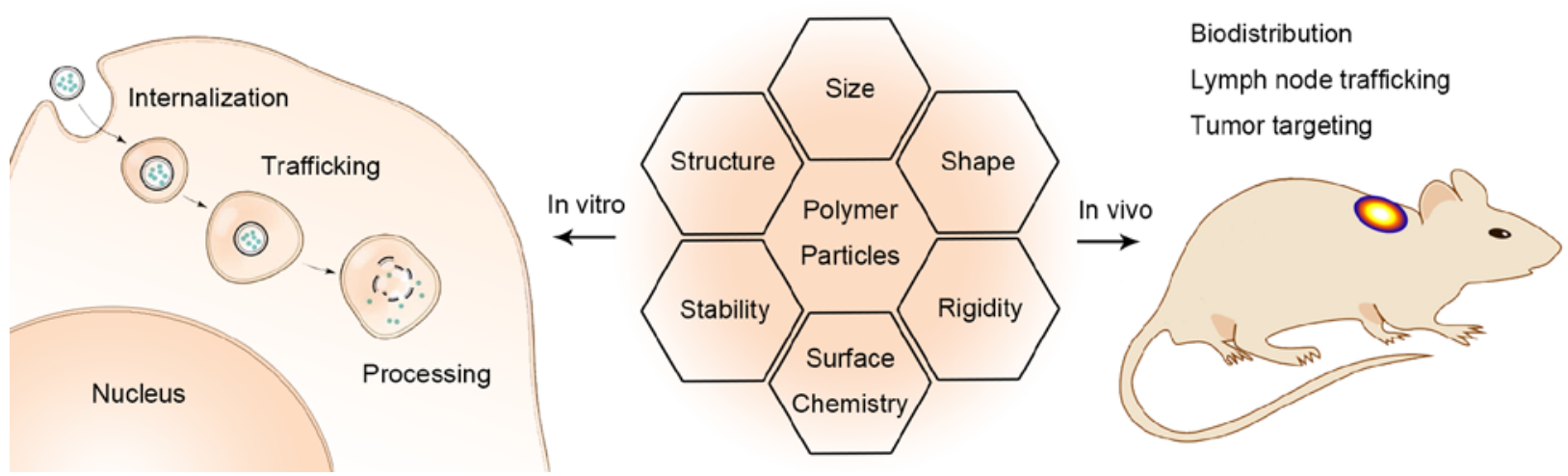
Figure 2. A summary of polymer particles with controlled size, shape, rigidity, surface chemistry, stability, and structure that have been explored for bio-nano interaction in vitro and in vivo.

Cellular association is not only governed by the physical properties of particles, but can also be tuned by varying the chemistry of the particle surface. For example, polymer particles composed of low-fouling materials (e.g., PEG) or modified with low-fouling polymers show decreased cellular association. ${ }^{32-34}$ To increase specific cellular binding or uptake, targeting molecules can be functionalized onto polymer particle surfaces either by electrostatic adsorption or covalent conjugation. ${ }^{17,18,22,34,35}$ Particles are exposed to a complex environment in cell cultures and in vivo, and without low-fouling surfaces, they will be rapidly coated with various biomolecules. This coating, termed the protein corona, is strongly influenced by particle size and surface chemistry. ${ }^{36}$ Furthermore, the protein corona can dramatically alter the bio-nano interactions depending on targeting ligand and cell type. ${ }^{23,36-38}$ Interestingly, targeting to cells increases association, but this may not actually increase uptake, which is highly dependent on the receptors targeted. $^{22,39}$ It is important to draw a distinction between "particles binding to the surface of cells" and "particles internalized by cells” (cellular uptake); cellular association includes both of these.

The cellular uptake mechanism (e.g., phagocytosis, macropinocytosis, clathrin-mediated endocytosis, caveolae-mediated endocytosis, and clathrin/caveolae-independent pathways) is influenced by particle properties, and this can vastly impact on biological outcomes. ${ }^{26}$ Cellular uptake is a time-dependent process, and longer incubation times result in higher numbers of 
internalized particles. ${ }^{20}$ Internalized particles are usually transported from endosomes to lysosomes in a matter of minutes, ${ }^{40}$ and less stiff particles have demonstrated faster transport to lysosomes. ${ }^{28}$ Interestingly, polymer particles located in the lysosome are not distributed equally between the two daughter cells upon cell division, with ubiquitylation playing a central role in the overall cell response to particles. ${ }^{40}$ Although hollow polymer particles with different aspect ratios or rigidities can have different uptake kinetics, these differences do not appear to influence intracellular fate. ${ }^{10,41}$ Combined with flow cytometry, polymer particles equipped with a switch that responds to microenvironmental changes in $\mathrm{pH}$ allows for sensitive, high-throughput investigation of particle-cell interactions in vitro. ${ }^{42}$

The reason for studying physicochemical effects on particle association and trafficking is both to increase our understanding and also to engineer particles for specific in vivo applications. In terms of biodistribution, smaller poly(ethylene glycol) (PEG) particles composed of large molecular weight PEG demonstrate extended in vivo circulation time when compared to larger particles and particles with lower molecular weight PEG. ${ }^{7}$ PEG particles without templates show significantly higher blood retention compared to MS@PEG particles with mesoporous silica templates. This may be because of differences in particle stiffness, as softer particles could minimize the filtration effect experienced during circulation in vivo by compressing similar to red blood cells. ${ }^{24,31,43}$ Another example of physicochemical properties affecting function is the observation that PEGylating polymer particles dramatically improves their lymphatic drainage in vivo. ${ }^{25}$ Still, there is a large gap between in vitro predictions and in vivo behavior. A compromise may be ex vivo: assays based on whole human blood are more predictive of PEG particle behavior in vivo than traditional cell-line based in vitro assays. ${ }^{7}$ 


\section{BIODEGRADATION}

One crucial material requirement of therapeutic polymer particles is the capacity to degrade or disassemble to release the encapsulated cargo via biological stimuli in targeted microenvironments (Figure 3). During cellular internalization, particles will often be exposed to a decrease in $\mathrm{pH}$, an increase in the amount of reducing agents, and an increase in enzyme concentration as they proceed from the extracellular fluid into the endosomes/lysosomes. ${ }^{26}$ The acidification of the endosome/lysosome can occur minutes after internalization, leading to a $\mathrm{pH}$ of $\sim 4-6$. Similarly, various enzymes are pumped into the endosome, lysosome or cytoplasm to further degrade and destroy both foreign and natural biological materials. Finally, cells also contain reducing agents, such as glutathione (GSH), that break disulphide bonds. These three biological degradation mechanisms ( $\mathrm{pH}$, enzymes, reducing agents) allow for a broad spectrum of engineered responses in polymer particles, though other less common triggers, such as sugar gradients ${ }^{44}$ or shear, ${ }^{45}$ also exist. 
A Examples of extracellular microenvironments

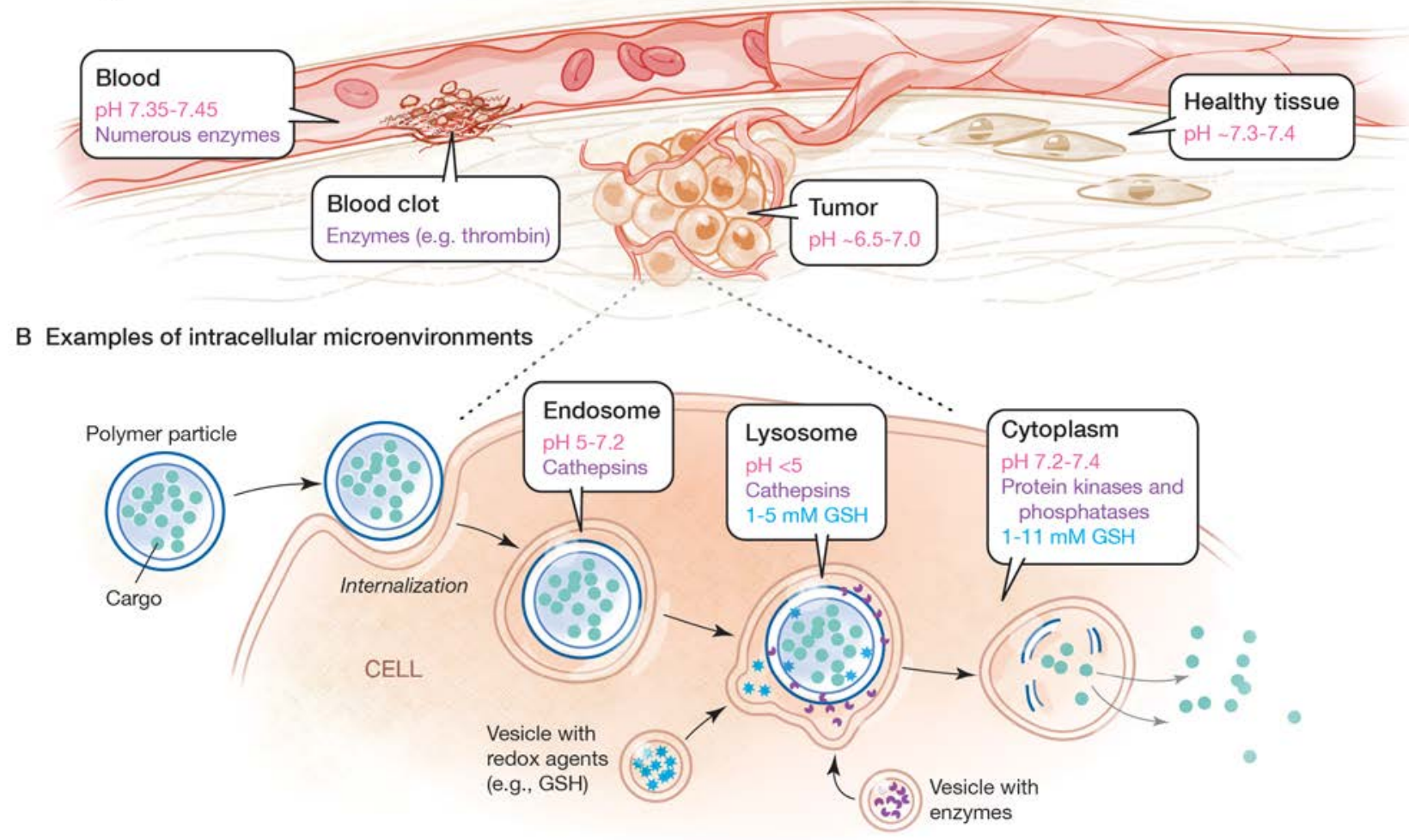

C Examples of responsive polymer particle design
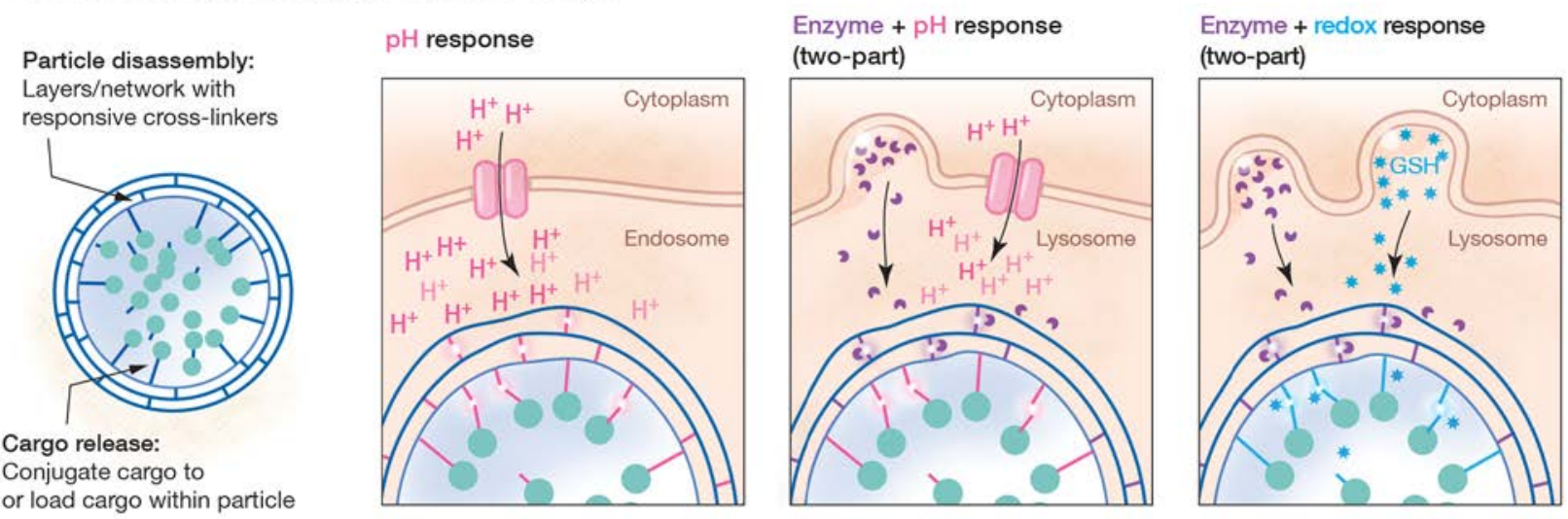

Figure 3. Examples of (A) extracellular and (B) intracellular microenvironments. (C) Rational design of responsive polymer particles and mechanisms of triggered particle degradation and/or cargo release via biological stimuli (e.g., $\mathrm{pH}$, enzyme, and redox) after internalization. The examples and values included are intended to provide a general overview and are not exhaustive. 
As polymer particles are internalized, the $\mathrm{pH}$ drops from 7.4 in the extracellular environment to a range of $\sim 6.5-4.5$ in endosomes or lysosomes. Therefore, materials that are either protonated or deprotonated in this range are promising candidates for engineering degradable particles. Synthetic polymers with a $K_{\mathrm{a}}$ below 7.4, such as poly(2-diisopropylaminoethyl methacrylate) (PDPA), are useful for forming $\mathrm{pH}$ responsive, degradable polymer particles. $^{46-48}$ Similarly, biotin-modified PEG can be used as a capping polymer to form neutravidin-iminobiotin bonds that rapidly decompose around $\mathrm{pH} 4-6$, as a result of the lower affinity of the protonated iminobiotin to neutravidin. ${ }^{49}$ Additionally, $\mathrm{pH}$ labile cross-linkers, such as 8-arm-PEGmethylmaleic anhydride, can be used to form protein replica particles capable of disassembling below pH 6.5 and after cellular internalization. ${ }^{50}$

Unlike synthetic polymers, the multiple charge states of both polyphenols (e.g., tannic acid, TA), and metal ions allow for metal-phenolic network (MPN) particles to exhibit varied $\mathrm{pH}$ disassembly profiles. ${ }^{51,52}$ Generally, MPN particles composed of higher valence metal ions are more stable under acidic $\mathrm{pH}$ than those composed of lower valence metal ions $\left(\mathrm{Zr}^{4+}>\mathrm{Al}^{3+}>\mathrm{Cu}^{2+}\right)$. Interestingly, $\mathrm{Fe}^{3+}$ results in one of the most stable MPN particles, likely due to specific affinity differences between polyphenols and certain metal ions. $\mathrm{Al}^{3+}$ has been shown to have favorable disassembly kinetics for drug delivery, and can degrade and release anticancer drugs in the endosome/lysosome. ${ }^{53}$ Polymer-phenol conjugates can also form MPNs, which allow for the

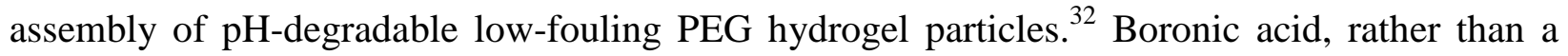
metal, can be combined with TA to form boronate-phenolic network (BPN) particles that are responsive to both $\mathrm{pH}$ and sugar. ${ }^{44}$ 
Polymer particles composed of biomolecules or containing biomolecule cross-linkers can be degraded by enzymes encountered in the bloodstream and during cellular internalization. Hollow particles composed solely of proteins are promising candidates for protease degradable carriers, ${ }^{12}$ and the chemical properties of the proteins (reduced, native, or oxidized) can play a role in their degradation profiles. ${ }^{54}$ Similarly, particles containing or composed of poly(L-lysine), poly(Lglutamic acid), poly(L-arginine), poly(L-histidine), or gelatin can also be degraded by proteases in solution and in the proteolytic environment of endosomes/lysosomes. ${ }^{55-60}$ By cross-linking synthetic polymer particles with an enzyme-cleavable peptide sequences, degradation can be engineered in response to specific proteases (e.g., cathepsin B or thrombin), allowing for in vitro degradation within cancer cells, ${ }^{61}$ or in the thrombus microenvironment. ${ }^{62}$

The intracellular environment contains reducing agents (e.g., GSH), which can help to degrade polymer particles stabilized with disulfide bonds. Disulfide bonds are usually assembled by cross-linking polymers with a disulfide cross-linker, or by cross-linking thiolated polymers using oxidizing reagents or thiol-disulfide exchange..$^{9,21,48,63}$ Cargo can also be conjugated to particles via disulfide bonds, and can then be released in the presence of reducing agents after internalization. ${ }^{64}$ However, the majority of polymer particles are internalized into acidic compartments within the cell, where redox-responsive materials are less effective. ${ }^{47}$ Therefore, designing responsive particles effective at lower $\mathrm{pH}$ is of interest. To achieve this, dualresponsive hollow LbL polymer particles - using the synergistic effects of $\mathrm{pH}$ and redoxpotential - can be engineered which allow for rapid and efficient cargo release, even at extremely low concentrations of $\mathrm{GSH}^{46}$ In addition, the degradation rates in both simulated cellular conditions and in cells can be finely tuned by varying the amount of disulfide cross-linker for redox sensitive particles. ${ }^{48}$ 


\section{BIOMEDICAL APPLICATIONS}

Our increasing ability to engineer polymer particles with specific properties, combined with our increasing understanding of their bio-nano interactions, has provided the impetus for studying their application within biomedicine. Example applications that have been investigated for polymer particles include: (i) drug and gene delivery, (ii) vaccination and immunostimulation, (iii) sensing of bacteria and viruses, and (iv) confined bioreactions (Figure 4).

The advantage of using templated polymer particles for drug and gene delivery is the high level of control that can be exerted over particle properties. A drug carrier can deliver and release a drug in response to changes in redox potential, $\mathrm{pH}$, or enzyme concentration: multiple templated particle systems have successfully demonstrated intracellular delivery of therapeutics (Figure 4A). Examples include hollow or porous polymer particles with either hydrophobic drugs (e.g., paclitaxel and thiocoraline) or with hydrophilic drugs (e.g., doxorubicin) conjugated to the polymers used to assemble the particles. ${ }^{13,63,65,66}$ One of these particles has demonstrated the ability to circumvent drug resistance mechanisms in multi-drug resistant cancer cells. ${ }^{67}$ Protein therapeutics can also be embedded in the capsule during LbL assembly, and these particles have been used to induce bone formation in mice with the help of embryonic stem cells. ${ }^{68}$ In addition, hollow polymer particles loaded with enzymes (e.g., urokinase plasminogen activator) have been used to target activated platelets and release the cargo for fibrin degradation in the presence of thrombin. ${ }^{62}$ Intracellular delivery of drugs can also be performed using polydopamine particles modified with a drug-polymer conjugate, or by using MPN particles assembled on drug-loaded calcium carbonate templates. ${ }^{53,69}$ Both of these systems demonstrate $\mathrm{pH}$-responsive drug release 
with cytotoxicity at similar, and even lower, concentrations than free drug. Gene delivery with resulting gene silencing can be achieved using a MS templating technique wherein albumin or polypeptide particles are used to deliver small interfering RNA (siRNA) ${ }^{11,57}$ 
A Drug and gene delivery

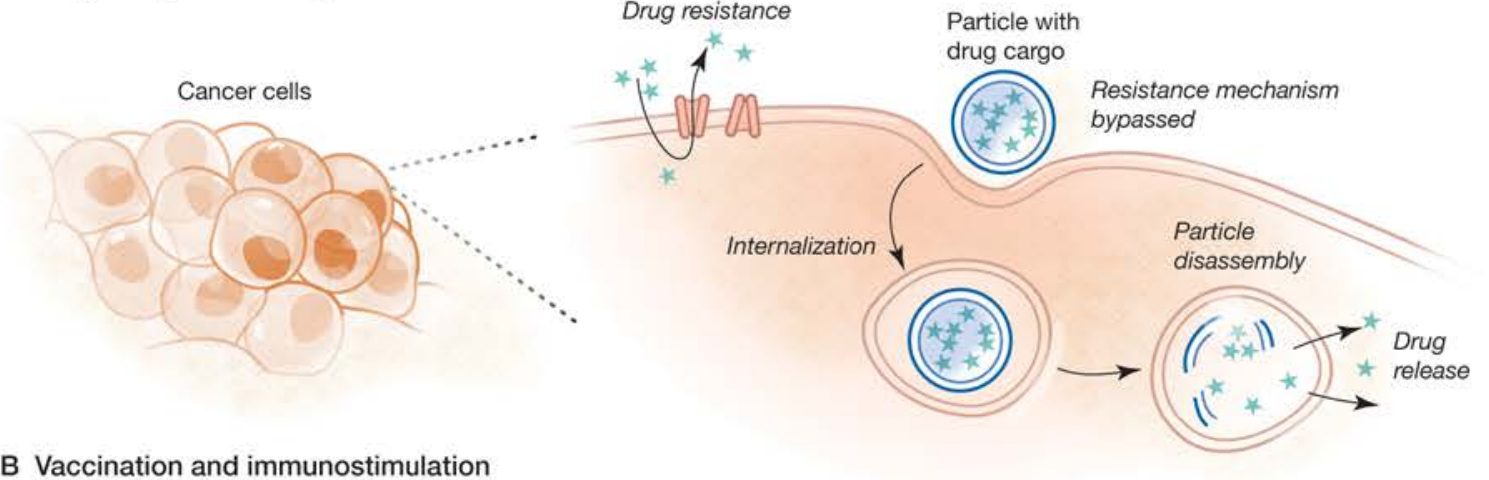

B Vaccination and immunostimulation

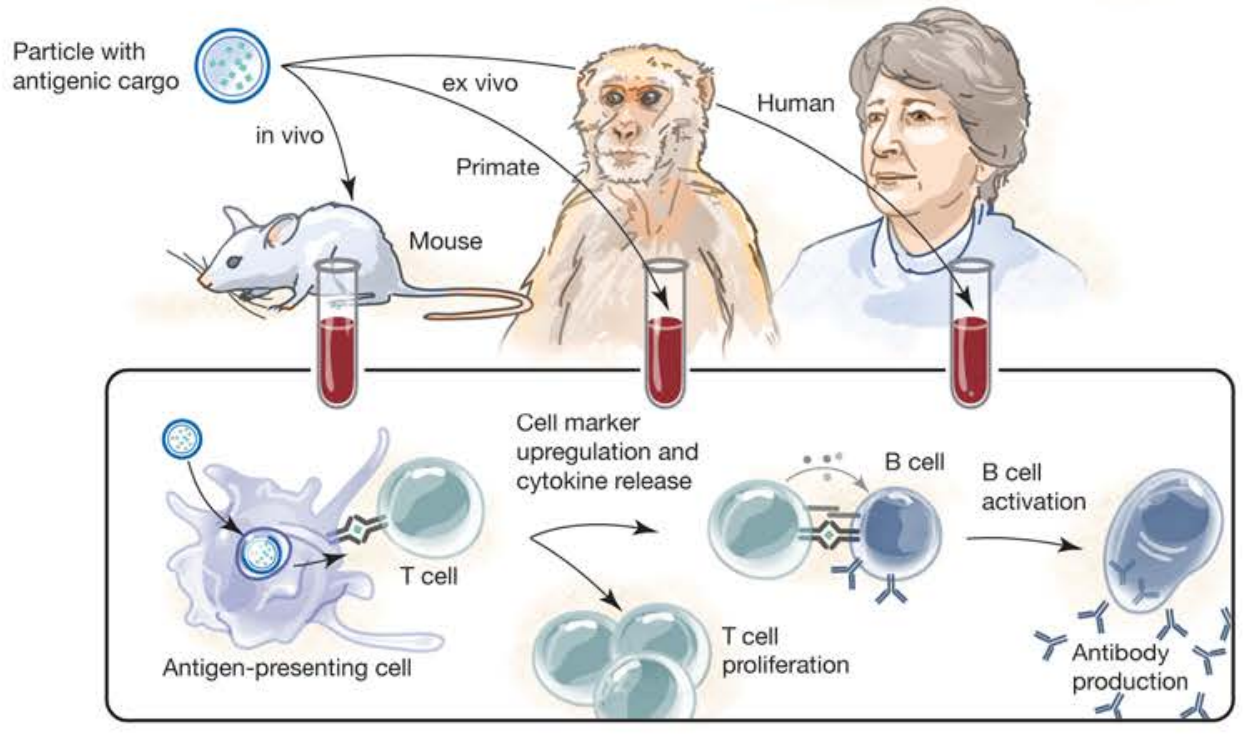

\section{Biosensing}

Detection and differentiation sensor
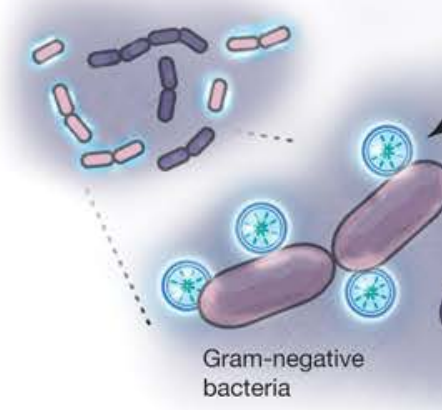

\section{Confined bioreactions}

\section{Localized reactions}

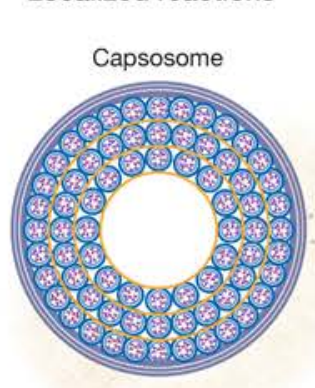

Particle filled with liquid crystals

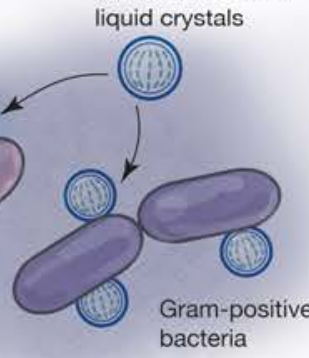
bacteria
Internalization sensor

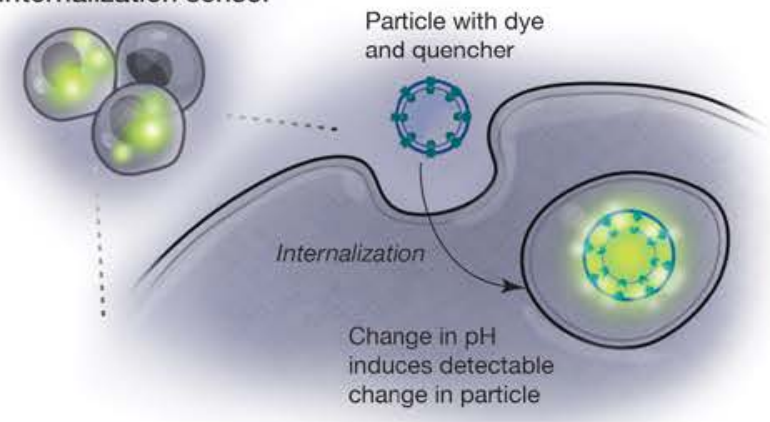

Encapsulated reactions

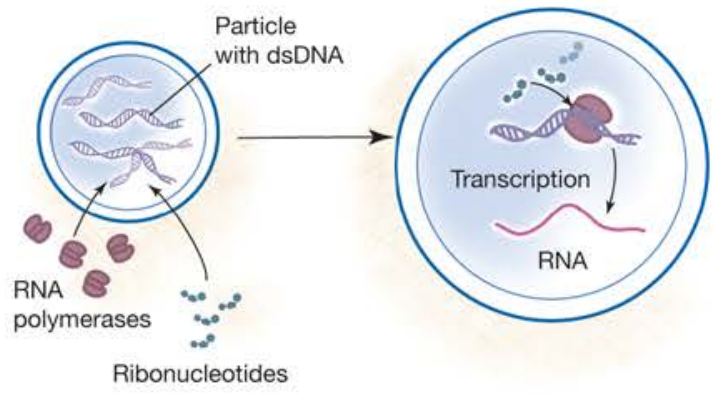


Figure 4. Potential applications of polymer particles include: (A) drug and gene delivery, (B) vaccination and immunostimulation, (C) biosensing, and (D) confined bioreactions.

Templated polymer particles are additionally of interest for immunostimulation and vaccination, as the immune system inherently recognizes, investigates, and processes exogenous particles in the size range of viruses and bacteria (Figure 4B). ${ }^{70}$ For example, hollow LbL polymer particles loaded with antigens (e.g., KP9 or ovalbumin) can be processed by antigen presenting cells (APCs) to activate antigen-specific $\mathrm{T}$ cells. ${ }^{64,71}$ MS-mediated ovalbumin particles and selfadjuvanting polypeptide particles conjugated with $\mathrm{CpG}$ have been used for $\mathrm{T}$ cell response in immunized mice and human dendritic cell activation, respectively. ${ }^{9,54}$ LbL capsules with different shapes can result in different degrees of cytokine secretion. ${ }^{72}$ In addition, surface engineering of polymer particles with PEGylation not only improves their lymphatic drainage but also increases the priming of antigen-specific $\mathrm{T}$ cells in vivo. ${ }^{25}$ Finally, nanoengineered synthetic vaccine particles can mimic microbial structures and functions to induce protective immunity against cancer and viral infection. ${ }^{73}$

Sensors used to detect and report changes in their environment can also be assembled through templating (Figure 4C). Hollow LbL polymer particles can be backfilled with liquid crystal emulsions followed by disassembly of the templating particles. When these liquid crystals come into contact with Gram negative bacteria or lipid-enveloped viruses they transition from a bipolar to a radial configuration, a change that is easy to observe with polarized light. ${ }^{74}$ This transition is not seen for Gram positive bacteria or non-enveloped viruses, and can therefore be used to distinguish between different types of bacteria and viruses. For probing carrier internalization by 
cells, current technologies are limited by either low throughput or low sensitivity due to the nature of the fluorescent probes. Hollow LbL particles with a fluorescence probe that switches "on" and "off", depending on the pH, can be used to probe endocytosis (Figure 4C). ${ }^{42}$ These hollow particles are assembled with a fluorescent dye and a quencher incorporated into the multilayers. At extracellular $\mathrm{pH}$ the dye and quencher are close to each other and the fluorescence is quenched. At lower (intracellular) $\mathrm{pH}$ the particles swell and the quenching is lost, which leads to recovery of fluorescence.

Templated polymer particles can be used as bioreactors, for example using biomimetic multicompartmentalized colloidal assemblies (Figure 4D). ${ }^{75}$ This concept is inspired by the structure of biological cells, where reactions are highly controlled both spatially and temporally with the help of compartmentalization. Triggered enzymatic degradation of DNA or continuous synthesis of RNA can both be performed inside hollow polymer particles. ${ }^{76,77}$ Using a multi-level hierarchical structure of liposomes assembled between layers in a hollow LbL particle, so called “capsosomes" can be made. ${ }^{78}$ By exploiting temperature-induced changes in permeability of the liposomal membranes an enzymatic reaction can be repeatedly initiated, with the enzyme remaining trapped inside the assembly, while the permeability of the substrate through the lipid membranes is controlled. ${ }^{78}$

\section{CONCLUSION AND PERSPECTIVES}

In this Account, we have discussed our recent advances in the application of a range of templated polymer particles toward the study and exploitation of bio-nano interactions, including cellular binding and uptake, intracellular trafficking, and biodistribution. The study of bio-nano 
interactions integrates biological and chemical knowledge, not only to help inspire new materials, but also to provide fundamentals that can revolutionize our understanding of therapeutic delivery. The goal of this area is to design and understand particle systems that can negotiate biological barriers, thus increasing therapeutic efficacy in disease processes ranging from inflammation to cancer. The interdependent, and sometimes contradictory, roles that particle size, shape, rigidity, structure, and surface chemistry have on the cellular internalization, intracellular trafficking, biocompatibility, and biodistribution behavior must also be considered (Figure 5). For example, combining stealth (reduced non-specific interactions with healthy tissues) with targeting (increased specific interactions with diseased tissues) is desirable, but particle properties responsible for these end-behaviors are often incongruous. Another example of this phenomenon is the compromise between stable drug-encapsulation in robustly assembled particles contrasted with the controlled release of drugs from easily degradable particles. Further complicating design choices is the challenge of determining which physicochemical properties are responsible for a particular in vivo behavior. Deliberate particle design requires a thorough understanding of the underlying bio-nano interactions. Improving our understanding in this area will therefore open new opportunities and enable innovations in the fields of material science, biology and nanobiotechnology. In addition, we contribute a free web-based tool to organize and share our on-going journey in the research of bio-nano interactions, ${ }^{79}$ and hope that this can inspire other research groups to do something similar. We believe this to have great potential in facilitating the dissemination, organization and retrieval of information in this complex field and thus accelerate the development of new and improved polymer particles, with well-understood and controlled bio-nano interactions. 


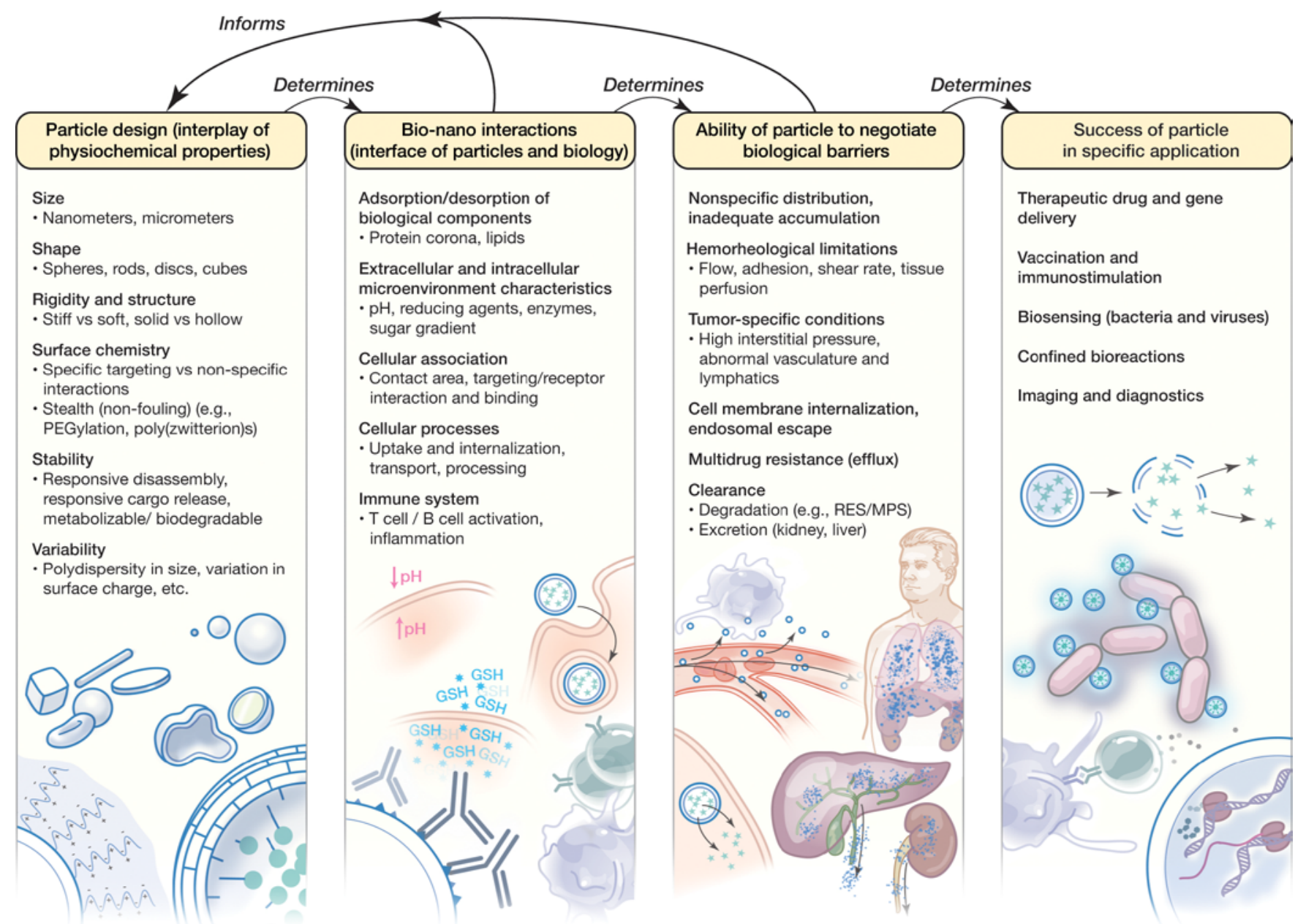

Abbreviations: PEG indicates poly(ethylene glycol); MPS, mononuclear phagocyte system; RES, reticuloendothelial cell system

Figure 5. The interplay of physicochemical properties of polymer particles not only influences their compatibility, distribution, and ability to overcome biological barriers to delivery and effectiveness, but also determines their potential applications. Rather than attempting to solely design the ultimate ideal particle, purposeful particle design should seek to understand the nature of relevant bio-nano interactions, and account for the tradeoffs and interdependencies in particle properties. The eventual success of a particle for a particular application can be enhanced by allowing these factors to inform upstream engineering and manufacturing decisions. The examples included are intended to provide a general overview and are not exhaustive. 


\section{AUTHOR INFORMATION}

\section{Corresponding Author}

*E-mail: fcaruso@unimelb.edu.au

\section{Notes}

The authors declare no competing financial interest.

\section{Biographical Information}

Jiwei Cui received his Ph.D. in colloid and interface chemistry from Shandong University in 2010. He is currently a research fellow in the Department of Chemical and Biomolecular Engineering at the University of Melbourne. His research interests include interface engineering, particle assembly and therapeutic delivery.

Joseph J. Richardson received his Ph.D. in chemical and biomolecular engineering from the University of Melbourne in 2015. He is currently an OCE Fellow at the Commonwealth Science and Industry Research Organization (CSIRO) in Australia, and is interested in developing novel polymer assembly techniques and applying old technologies to new biomedical challenges.

Mattias Björnmalm received his M.S. in engineering nanoscience from Lund University in 2012. He is currently pursuing a Ph.D. at the University of Melbourne under the supervision of Professor Frank Caruso.

Matthew Faria received his B.S. in computer science from Carnegie Mellon University in 2008. He is currently pursuing a Ph.D. at the University of Melbourne under the supervision of Professors Frank Caruso and Edmund Crampin. His research focus is in using computational modelling to understand interactions between nanomaterials and biological systems. 
Frank Caruso is a professor and Australian Research Council Australian Laureate Fellow at the University of Melbourne. He received his Ph.D. in 1994 from the University of Melbourne. His research interests focus on developing advanced nano- and biomaterials for biotechnology and medicine.

\section{ACKNOWLEDGMENT}

This work was supported by the ARC under the Australian Laureate Fellowship (FL120100030, F.C.) and through the ARC Centre of Excellence in Convergent Bio-Nano Science and Technology (project number CE140100036). J.J.R acknowledges the OCE Scheme.

\section{REFERENCES}

(1) Nel, A. E.; Mädler, L.; Velegol, D.; Xia, T.; Hoek, E. M.; Somasundaran, P.; Klaessig, F.; Castranova, V.; Thompson, M. Understanding Biophysicochemical Interactions at the Nano-Bio Interface. Nat. Mater. 2009, 8, 543-557.

(2) Mitragotri, S.; Burke, P. A.; Langer, R. Overcoming the Challenges in Administering Biopharmaceuticals: Formulation and Delivery Strategies. Nat. Rev. Drug Discov. 2014, 13, 655-672.

(3) Petros, R. A.; DeSimone, J. M. Strategies in the Design of Nanoparticles for Therapeutic Applications. Nat. Rev. Drug Discov. 2010, 9, 615-627.

(4) Petrosko, S. H.; Fromen, C. A.; Auyeung, E.; DeSimone, J. M.; Mirkin, C. A. Nanotechnology: An Enduring Bridge between Engineering and Medicine. The Bridge 2013, 43, 7-15.

(5) Wang, J.; Byrne, J. D.; Napier, M. E.; DeSimone, J. M. More Effective Nanomedicines through Particle Design. Small 2011, 7, 1919-1931.

(6) Albanese, A.; Tang, P. S.; Chan, W. C. The Effect of Nanoparticle Size, Shape, and Surface Chemistry on Biological Systems. Annu. Rev. Biomed. Eng. 2012, 14, 1-16.

(7) Cui, J.; De Rose, R.; Alt, K.; Alcantara, S.; Paterson, B. M.; Liang, K.; Hu, M.; Richardson, J. J.; Yan, Y.; Jeffery, C. M.; Price, R. I.; Peter, K.; Hagemeyer, C. E.; Donnelly, P. S.; Kent, S. J.; Caruso, F. Engineering Poly(ethylene glycol) Particles for Improved Biodistribution. ACS Nano 2015, 9, 1571-1580.

(8) Shimoni, O.; Yan, Y.; Wang, Y.; Caruso, F. Shape-Dependent Cellular Processing of Polyelectrolyte Capsules. ACS Nano 2013, 7, 522-530.

(9) Cui, J.; De Rose, R.; Best, J. P.; Johnston, A. P. R.; Alcantara, S.; Liang, K.; Such, G. K.; Kent, S. J.; Caruso, F. Mechanically Tunable, Self-Adjuvanting Nanoengineered Polypeptide Particles. Adv. Mater. 2013, 25, 3468-3472.

(10) Sun, H.; Wong, E. H.; Yan, Y.; Cui, J.; Dai, Q.; Guo, J.; Qiao, G. G.; Caruso, F. The Role of Capsule Stiffness on Cellular Processing. Chem. Sci. 2015, 6, 3505-3514. 
(11) Mertz, D.; Affolter-Zbaraszczuk, C.; Barthes, J.; Cui, J.; Caruso, F.; Baumert, T. F.; Voegel, J. C.; Ogier, J.; Meyer, F. Templated Assembly of Albumin-Based Nanoparticles for Simultaneous Gene Silencing and Magnetic Resonance Imaging. Nanoscale 2014, 6, 1167611680.

(12) Mertz, D.; Wu, H.; Wong, J. S.; Cui, J.; Tan, P.; Alles, R.; Caruso, F. Ultrathin, Bioresponsive and Drug-Functionalized Protein Capsules. J. Mater. Chem. 2012, 22, 2143421442.

(13) Wang, Y.; Bansal, V.; Zelikin, A. N.; Caruso, F. Templated Synthesis of SingleComponent Polymer Capsules and Their Application in Drug Delivery. Nano Lett. 2008, 8, 1741-1745.

(14) Richardson, J. J.; Björnmalm, M.; Caruso, F. Technology-Driven Layer-by-Layer Assembly of Nanofilms. Science 2015, 348, aaa2491.

(15) Cui, J.; Wang, Y.; Postma, A.; Hao, J.; Hosta-Rigau, L.; Caruso, F. Monodisperse Polymer Capsules: Tailoring Size, Shell Thickness, and Hydrophobic Cargo Loading via Emulsion Templating. Adv. Funct. Mater. 2010, 20, 1625-1631.

(16) Wang, Y.; Yu, A.; Caruso, F. Nanoporous Polyelectrolyte Spheres Prepared by Sequentially Coating Sacrificial Mesoporous Silica Spheres. Angew. Chem. Int. Ed. 2005, 117, 2948-2952.

(17) Cortez, C.; Tomaskovic-Crook, E.; Johnston, A. P. R.; Radt, B.; Cody, S. H.; Scott, A. M.; Nice, E. C.; Heath, J. K.; Caruso, F. Targeting and Uptake of Multilayered Particles to Colorectal Cancer Cells. Adv. Mater. 2006, 18, 1998-2003.

(18) Cortez, C.; Tomaskovic-Crook, E.; Johnston, A. P. R.; Scott, A. M.; Nice, E. C.; Heath, J. K.; Caruso, F. Influence of Size, Surface, Cell Line, and Kinetic Properties on the Specific Binding of A33 Antigen-Targeted Multilayered Particles and Capsules to Colorectal Cancer Cells. ACS Nano 2007, 1, 93-102.

(19) De Rose, R.; Zelikin, A. N.; Johnston, A. P. R.; Sexton, A.; Chong, S.-F.; Cortez, C.; Mulholland, W.; Caruso, F.; Kent, S. J. Binding, Internalization, and Antigen Presentation of Vaccine-Loaded Nanoengineered Capsules in Blood. Adv. Mater. 2008, 20, 4698-4703.

(20) Yan, Y.; Johnston, A. P. R.; Dodds, S. J.; Kamphuis, M. M.; Ferguson, C.; Parton, R. G.; Nice, E. C.; Heath, J. K.; Caruso, F. Uptake and Intracellular Fate of Disulfide-Bonded Polymer Hydrogel Capsules for Doxorubicin Delivery to Colorectal Cancer Cells. ACS Nano 2010, 4, 2928-2936.

(21) Yan, Y.; Wang, Y.; Heath, J. K.; Nice, E. C.; Caruso, F. Cellular Association and Cargo Release of Redox-Responsive Polymer Capsules Mediated by Exofacial Thiols. Adv. Mater. 2011, 23, 3916-3921.

(22) Johnston, A. P. R.; Kamphuis, M. M.; Such, G. K.; Scott, A. M.; Nice, E. C.; Heath, J. K.; Caruso, F. Targeting Cancer Cells: Controlling the Binding and Internalization of Antibody-Functionalized Capsules. ACS Nano 2012, 6, 6667-6674.

(23) Yan, Y.; Gause, K. T.; Kamphuis, M. M.; Ang, C.-S.; O’Brien-Simpson, N. M.; Lenzo, J. C.; Reynolds, E. C.; Nice, E. C.; Caruso, F. Differential Roles of the Protein Corona in the Cellular Uptake of Nanoporous Polymer Particles by Monocyte and Macrophage Cell Lines. ACS Nano 2013, 7, 10960-10970.

(24) Cui, J.; Björnmalm, M.; Liang, K.; Xu, C.; Best, J. P.; Zhang, X.; Caruso, F. Super-Soft Hydrogel Particles with Tunable Elasticity in a Microfluidic Blood Capillary Model. Adv. Mater. 2014, 26, 7295-7299. 
(25) De Koker, S.; Cui, J.; Vanparijs, N.; Albertazzi, L.; Grooten, J.; Caruso, F.; De Geest, B. G. Engineering Polymer Hydrogel Nanoparticles for Lymph Node Targeted Delivery. Angew. Chem. Int. Ed. 2016, 55, 1334-1339.

(26) Yan, Y.; Such, G. K.; Johnston, A. P. R.; Best, J. P.; Caruso, F. Engineering Particles for Therapeutic Delivery: Prospects and Challenges. ACS Nano 2012, 6, 3663-3669.

(27) Blanco, E.; Shen, H.; Ferrari, M. Principles of Nanoparticle Design for Overcoming Biological Barriers to Drug Delivery. Nat. Biotechnol. 2015, 33, 941-951.

(28) Hartmann, R.; Weidenbach, M.; Neubauer, M.; Fery, A.; Parak, W. J. StiffnessDependent in Vitro Uptake and Lysosomal Acidification of Colloidal Particles. Angew. Chem. Int. Ed. 2015, 54, 1365-1368.

(29) Anselmo, A. C.; Mitragotri, S. Impact of Particle Elasticity on Particle-Based Drug Delivery Systems. Adv. Drug. Deliv. Rev. 2016, DOI: 10.1016/j.addr.2016.01.007.

(30) Mertz, D.; Cui, J.; Yan, Y.; Devlin, G.; Chaubaroux, C.; Dochter, A.; Alles, R.; Lavalle, P.; Voegel, J. C.; Blencowe, A. Protein Capsules Assembled via Isobutyramide Grafts: Sequential Growth, Biofunctionalization, and Cellular Uptake. ACS Nano 2012, 6, 7584-7594.

(31) Anselmo, A. C.; Zhang, M.; Kumar, S.; Vogus, D. R.; Menegatti, S.; Helgeson, M. E.; Mitragotri, S. Elasticity of Nanoparticles Influences Their Blood Circulation, Phagocytosis, Endocytosis, and Targeting. ACS Nano 2015, 9, 3169-3177.

(32) Ju, Y.; Cui, J.; Müllner, M.; Suma, T.; Hu, M.; Caruso, F. Engineering LowFouling and $\mathrm{pH}$-Degradable Capsules through the Assembly of Metal-Phenolic Networks. Biomacromolecules 2015, 16, 807-814.

(33) Müllner, M.; Cui, J.; Noi, K. F.; Gunawan, S. T.; Caruso, F. Surface-Initiated Polymerization within Mesoporous Silica Spheres for the Modular Design of Charge-Neutral Polymer Particles. Langmuir 2014, 30, 6286-6293.

(34) Leung, M. K.; Hagemeyer, C. E.; Johnston, A. P. R.; Gonzales, C.; Kamphuis, M. M.; Ardipradja, K.; Such, G. K.; Peter, K.; Caruso, F. Bio-Click Chemistry: Enzymatic Functionalization of PEGylated Capsules for Targeting Applications. Angew. Chem. Int. Ed. 2012, 124, 7244-7248.

(35) Kamphuis, M. M.; Johnston, A. P. R.; Such, G. K.; Dam, H. H.; Evans, R. A.; Scott, A. M.; Nice, E. C.; Heath, J. K.; Caruso, F. Targeting of Cancer Cells Using ClickFunctionalized Polymer Capsules. J. Am. Chem. Soc. 2010, 132, 15881-15883.

(36) Monopoli, M. P.; Åberg, C.; Salvati, A.; Dawson, K. A. Biomolecular Coronas Provide the Biological Identity of Nanosized Materials. Nat. Nanotechnol. 2012, 7, 779-786.

(37) Dai, Q.; Yan, Y.; Ang, C.-S.; Kempe, K.; Kamphuis, M. M.; Dodds, S. J.; Caruso, F. Monoclonal Antibody-Functionalized Multilayered Particles: Targeting Cancer Cells in the Presence of Protein Coronas. ACS Nano 2015, 9, 2876-2885.

(38) Dai, Q.; Yan, Y.; Guo, J.; Björnmalm, M.; Cui, J.; Sun, H.; Caruso, F. Targeting Ability of Affibody-Functionalized Particles is Enhanced by Albumin but Inhibited by Serum Coronas. ACS Macro Lett. 2015, 4, 1259-1263.

(39) Mintern, J. D.; Percival, C.; Kamphuis, M. M.; Chin, W. J.; Caruso, F.; Johnston, A. P. R. Targeting Dendritic Cells: The Role of Specific Receptors in the Internalization of Polymer Capsules. Adv. Healthc. Mater. 2013, 2, 940-944.

(40) Yan, Y.; Lai, Z. W.; Goode, R. J.; Cui, J.; Bacic, T.; Kamphuis, M. M.; Nice, E. C.; Caruso, F. Particles on the Move: Intracellular Trafficking and Asymmetric Mitotic Partitioning of Nanoporous Polymer Particles. ACS Nano 2013, 7, 5558-5567. 
(41) Shimoni, O.; Yan, Y.; Wang, Y.; Caruso, F. Shape-Dependent Cellular Processing of Polyelectrolyte Capsules. ACS Nano 2012, 7, 522-530.

(42) Liang, K.; Gunawan, S. T.; Richardson, J. J.; Such, G. K.; Cui, J. W.; Caruso, F. Endocytic Capsule Sensors for Probing Cellular Internalization. Adv. Healthc. Mater. 2014, 3, 1551-1554.

(43) Sun, H.; Björnmalm, M.; Cui, J.; Wong, E. H. H.; Dai, Y.; Dai, Q.; Qiao, G. G.; Caruso, F. Structure Governs the Deformability of Polymer Particles in a Microfluidic Blood Capillary Model. ACS Macro Lett. 2015, 4, 1205-1209.

(44) Guo, J.; Sun, H.; Alt, K.; Tardy, B. L.; Richardson, J. J.; Suma, T.; Ejima, H.; Cui, J.; Hagemeyer, C. E.; Caruso, F. Boronate-Phenolic Network Capsules with Dual Response to Acidic $\mathrm{pH}$ and cis-Diols. Adv. Healthc. Mater. 2015, 4, 1796-1801.

(45) Korin, N.; Kanapathipillai, M.; Matthews, B. D.; Crescente, M.; Brill, A.; Mammoto, T.; Ghosh, K.; Jurek, S.; Bencherif, S. A.; Bhatta, D. Shear-Activated Nanotherapeutics for Drug Targeting to Obstructed Blood Vessels. Science 2012, 337, 738-742.

(46) Liang, K.; Such, G. K.; Zhu, Z.; Yan, Y.; Lomas, H.; Caruso, F. Charge-Shifting Click Capsules with Dual-Responsive Cargo Release Mechanisms. Adv. Mater. 2011, 23, H273H277.

(47) Liang, K.; Such, G. K.; Johnston, A. P. R.; Zhu, Z.; Ejima, H.; Richardson, J. J.; Cui, J.; Caruso, F. Endocytic pH-Triggered Degradation of Nanoengineered Multilayer Capsules. Adv. Mater. 2014, 26, 1901-1905.

(48) Liang, K.; Such, G. K.; Zhu, Z.; Dodds, S. J.; Johnston, A. P. R.; Cui, J.; Ejima, H.; Caruso, F. Engineering Cellular Degradation of Multilayered Capsules through Controlled Cross-Linking. ACS Nano 2012, 6, 10186-10194.

(49) Poon, Z.; Chang, D.; Zhao, X.; Hammond, P. T. Layer-by-Layer Nanoparticles with a pH-Sheddable Layer for in Vivo Targeting of Tumor Hypoxia. ACS Nano 2011, 5, 42844292.

(50) Suma, T.; Cui, J.; Müllner, M.; Ju, Y.; Guo, J.; Hu, M.; Caruso, F. Generalizable Strategy for Engineering Protein Particles with pH-Triggered Disassembly and Recoverable Protein Functionality. ACS Macro Lett. 2015, 4, 160-164.

(51) Ejima, H.; Richardson, J. J.; Liang, K.; Best, J. P.; van Koeverden, M. P.; Such, G. K.; Cui, J.; Caruso, F. One-Step Assembly of Coordination Complexes for Versatile Film and Particle Engineering. Science 2013, 341, 154-157.

(52) Guo, J.; Ping, Y.; Ejima, H.; Alt, K.; Meissner, M.; Richardson, J. J.; Yan, Y.; Peter, K.; von Elverfeldt, D.; Hagemeyer, C. E.; Caruso, F. Engineering Multifunctional Capsules through the Assembly of Metal-Phenolic Networks. Angew. Chem. Int. Ed. 2014, 53, 5546-5551.

(53) Ping, Y.; Guo, J.; Ejima, H.; Chen, X.; Richardson, J. J.; Sun, H. L.; Caruso, F. pH-Responsive Capsules Engineered from Metal-Phenolic Networks for Anticancer Drug Delivery. Small 2015, 11, 2032-2036.

(54) Gause, K. T.; Yan, Y.; Cui, J.; O'Brien-Simpson, N. M.; Lenzo, J. C.; Reynolds, E. C.; Caruso, F. Physicochemical and Immunological Assessment of Engineered Pure Protein Particles with Different Redox States. ACS Nano 2015, 9, 2433-2444.

(55) Richardson, J. J.; Maina, J. W.; Ejima, H.; Hu, M.; Guo, J.; Choy, M. Y.; Gunawan, S. T.; Lybaert, L.; Hagemeyer, C. E.; De Geest, B. G.; Caruso, F. Versatile Loading of Diverse Cargo into Functional Polymer Capsules. Adv. Sci. 2015, 2, 1400007. 
(56) Gunawan, S. T.; Kempe, K.; Such, G. K.; Cui, J.; Liang, K.; Richardson, J. J.; Johnston, A. P. R.; Caruso, F. Tuning Particle Biodegradation through Polymer-Peptide Blend Composition. Biomacromolecules 2014, 15, 4429-4438.

(57) Cavalieri, F.; Beretta, G. L.; Cui, J.; Braunger, J. A.; Yan, Y.; Richardson, J. J.; Tinelli, S.; Folini, M.; Zaffaroni, N.; Caruso, F. Redox-Sensitive PEG-Polypeptide Nanoporous Particles for Survivin Silencing in Prostate Cancer Cells. Biomacromolecules 2015, 16, 21682178.

(58) Ochs, C. J.; Such, G. K.; Yan, Y.; van Koeverden, M. P.; Caruso, F. Biodegradable Click Capsules with Engineered Drug-Loaded Multilayers. ACS Nano 2010, 4, 1653-1663.

(59) Rivera-Gil, P.; De Koker, S.; De Geest, B. G.; Parak, W. J. Intracellular Processing of Proteins Mediated by Biodegradable Polyelectrolyte Capsules. Nano Lett. 2009, 9, 4398-4402.

(60) Maina, J. W.; Cui, J.; Björnmalm, M.; Wise, A. K.; Shepherd, R. K.; Caruso, F. Mold-Templated Inorganic-Organic Hybrid Supraparticles for Codelivery of Drugs. Biomacromolecules 2014, 15, 4146-4151.

(61) Gunawan, S. T.; Liang, K.; Such, G. K.; Johnston, A. P. R.; Leung, M. K.; Cui, J.; Caruso, F. Engineering Enzyme-Cleavable Hybrid Click Capsules with a pH-Sheddable Coating for Intracellular Degradation. Small 2014, 10, 4080-4086.

(62) Gunawan, S. T.; Kempe, K.; Bonnard, T.; Cui, J.; Alt, K.; Law, L. S.; Wang, X.; Westein, E.; Such, G. K.; Peter, K.; Hagemeyer, C. E.; Caruso, F. Multifunctional ThrombinActivatable Polymer Capsules for Specific Targeting to Activated Platelets. Adv. Mater. 2015, 27, 5153-5157.

(63) Sivakumar, S.; Bansal, V.; Cortez, C.; Chong, S.-F.; Zelikin, A. N.; Caruso, F. Degradable, Surfactant-Free, Monodisperse Polymer-Encapsulated Emulsions as Anticancer Drug Carriers. Adv. Mater. 2009, 21, 1820-1824.

(64) Chong, S.-F.; Sexton, A.; De Rose, R.; Kent, S. J.; Zelikin, A. N.; Caruso, F. A Paradigm for Peptide Vaccine Delivery Using Viral Epitopes Encapsulated in Degradable Polymer Hydrogel Capsules. Biomaterials 2009, 30, 5178-5186.

(65) Cui, J.; Yan, Y.; Wang, Y.; Caruso, F. Templated Assembly of pH-Labile Polymer-Drug Particles for Intracellular Drug Delivery. Adv. Funct. Mater. 2012, 22, 4718-4723.

(66) Wang, Y.; Yan, Y.; Cui, J.; Hosta-Rigau, L.; Heath, J. K.; Nice, E. C.; Caruso, F. Encapsulation of Water-Insoluble Drugs in Polymer Capsules Prepared Using Mesoporous Silica Templates for Intracellular Drug Delivery. Adv. Mater. 2010, 22, 4293-4297.

(67) Yan, Y.; Ochs, C. J.; Such, G. K.; Heath, J. K.; Nice, E. C.; Caruso, F. Bypassing Multidrug Resistance in Cancer Cells with Biodegradable Polymer Capsules. Adv. Mater. 2010, 22, 5398-5403.

(68) Facca, S.; Cortez, C.; Mendoza-Palomares, C.; Messadeq, N.; Dierich, A.; Johnston, A. P. R.; Mainard, D.; Voegel, J. C.; Caruso, F.; Benkirane-Jessel, N. Active Multilayered Capsules for in Vivo Bone Formation. Proc. Natl. Acad. Sci. U.S.A. 2010, 107, 3406-3411.

(69) Cui, J.; Yan, Y.; Such, G. K.; Liang, K.; Ochs, C. J.; Postma, A.; Caruso, F. Immobilization and Intracellular Delivery of an Anticancer Drug Using Mussel-Inspired Polydopamine Capsules. Biomacromolecules 2012, 13, 2225-2228. 
(70) De Koker, S.; Lambrecht, B. N.; Willart, M. A.; van Kooyk, Y.; Grooten, J.; Vervaet, C.; Remon, J. P.; De Geest, B. G. Designing Polymeric Particles for Antigen Delivery. Chem. Soc. Rev. 2011, 40, 320-339.

(71) Sexton, A.; Whitney, P. G.; Chong, S.-F.; Zelikin, A. N.; Johnston, A. P. R.; De Rose, R.; Brooks, A. G.; Caruso, F.; Kent, S. J. A Protective Vaccine Delivery System for in Vivo T Cell Stimulation Using Nanoengineered Polymer Hydrogel Capsules. ACS Nano 2009, 3, 3391-3400.

(72) Chen, X.; Yan, Y.; Müllner, M.; Ping, Y.; Cui, J.; Kempe, K.; Cortez-Jugo, C.; Caruso, F. Shape-Dependent Activation of Cytokine Secretion by Polymer Capsules in Human Monocyte-Derived Macrophages. Biomacromolecules 2016, 17, 1205-1212.

(73) De Geest, B. G.; Willart, M. A.; Hammad, H.; Lambrecht, B. N.; Pollard, C.; Bogaert, P.; De Filette, M.; Saelens, X.; Vervaet, C.; Remon, J. P. Polymeric Multilayer Capsule-Mediated Vaccination Induces Protective Immunity against Cancer and Viral Infection. ACS Nano 2012, 6, 2136-2149.

(74) Sivakumar, S.; Wark, K. L.; Gupta, J. K.; Abbott, N. L.; Caruso, F. Liquid Crystal Emulsions as the Basis of Biological Sensors for the Optical Detection of Bacteria and Viruses. Adv. Funct. Mater. 2009, 19, 2260-2265.

(75) Teo, B. M.; Hosta-Rigau, L.; Lynge, M. E.; Städler, B. Liposome-Containing Polymer Films and Colloidal Assemblies towards Biomedical Applications. Nanoscale 2014, 6, 6426-6433.

(76) Price, A. D.; Zelikin, A. N.; Wang, Y.; Caruso, F. Triggered Enzymatic Degradation of DNA within Selectively Permeable Polymer Capsule Microreactors. Angew. Chem. Int. Ed. 2009, 48, 329-332.

(77) Price, A. D.; Zelikin, A. N.; Wark, K. L.; Caruso, F. A Biomolecular "Ship-in-aBottle": Continuous RNA Synthesis within Hollow Polymer Hydrogel Assemblies. Adv. Mater. 2010, 22, 720-723.

(78) Chandrawati, R.; Hosta-Rigau, L.; Vanderstraaten, D.; Lokuliyana, S. A.; Städler, B.; Albericio, F.; Caruso, F. Engineering Advanced Capsosomes: Maximizing the Number of Subcompartments, Cargo Retention, and Temperature-Triggered Reaction. ACS Nano 2010, 4, 1351-1361.

(79) Bio-Nano

Research

Knowledgebase. http://bionano.eng.unimelb.edu.au/knowledge_base/ (Accessed May 2, 2016). 


\section{University Library}

\section{- M M N E R VA A gateway to Melbourne's research publications}

Minerva Access is the Institutional Repository of The University of Melbourne

Author/s:

Cui, J;Richardson, JJ;Bjornmalm, M;Faria, M;Caruso, F

Title:

Nanoengineered Templated Polymer Particles: Navigating the Biological Realm

Date:

2016-06-01

Citation:

Cui, J., Richardson, J. J., Bjornmalm, M., Faria, M. \& Caruso, F. (2016). Nanoengineered Templated Polymer Particles: Navigating the Biological Realm. ACCOUNTS OF CHEMICAL RESEARCH, 49 (6), pp.1139-1148. https://doi.org/10.1021/acs.accounts.6b00088.

Persistent Link:

http://hdl.handle.net/11343/108594 